\title{
BVG oder Säule 3a?
}

\section{Dank cleverer Planung früher in Pension gehen}

\section{Ausgangslage}

Wurde Ihre Vorsorgeplanung bereits durch einen Profi erstellt? Ja? Lesen Sie trotzdem kurz den folgenden Fall. Wir sind überzeugt, dass Ihre Planung noch weiter optimiert werden kann!

Dr. med. H. M. ist 49 Jahre alt und arbeitet seit einigen Jahren in seiner eigenen Praxis. Vor einiger Zeit hat er folgende Vorsorgelösungen abgeschlossen:

- Eine Pensionskassenlösung bei einer grossen Versicherung. Die Einkaufsmöglichkeiten sind vollständig ausgeschöpft.

- Ein gebundenes Vorsorgekonto Säule 3a mit einem Jahresbeitrag von CHF 6192 bei einer Schweizer Grossbank. Der aktuelle Zinssatz beträgt $1.25 \%$ p.a.

\section{Kein Verbesserungspotential vorhanden? Unsere Berater zeigen Ihnen den Weg!}

Folgende Verbesserungen konnten umgesetzt werden:

- Wechsel der Pensionskasse zu einer standeseigenen Organisation. Dadurch werden Einsparungen bei den Risiko- und Verwaltungskosten realisiert. Durch die Erhöhung der jährlichen Sparbeiträge werden die Altersleistungen verbessert und zusätzlich Steuern gespart.

- Dank der höheren Sparbeiträge werden neue Einkaufsmöglichkeiten geschaffen, womit wieder Steuern gespart werden können.

- Abschluss einer zusätzlichen Vorsorgepolice Säule 3a bei der Versicherung der Schweizer Ärzte. Dadurch wird die Steuerprogression bei der Auszahlung gebrochen. Zudem wird das Sparguthaben zu einem technischen Zinssatz von $2.25 \%$ (garantiert) verzinst.

Durch diese Optimierungen wird die jährliche Steuerbelastung um rund Fr. 10000 .reduziert.

\section{Profitieren Sie von unserem Angebot}

Ihre Bedürfnisse stehen bei uns im Mittelpunkt. Mit unserem Namen garantieren wir Ihnen die Betreuung durch ein ausgewiesenes Spezialistenteam mit langjähriger Erfahrung im Versicherungsund Bankenbereich. Dank unserer Unabhängigkeit können wir Ihnen das für Sie am besten geeignete Produkt anbieten.

\section{Antworttalon}

Vorname / Name

Adresse

PLZ / Ort

Geburtsdatum

Telefon Privat/Geschäft

Beste Zeit für einen Anruf

Ich wünsche eine persönliche Beratung. Bitte rufen Sie mich für einen unverbindlichen kostenlosen Ersttermin an.

Ich interessiere mich für folgende Produkte:

\footnotetext{
Pensionskasse BVG

Krankenkasse

Fondssparplan

Säule $3 a$

Rechtsschutzversicherung

Berufshaftpflichtversicherung
}

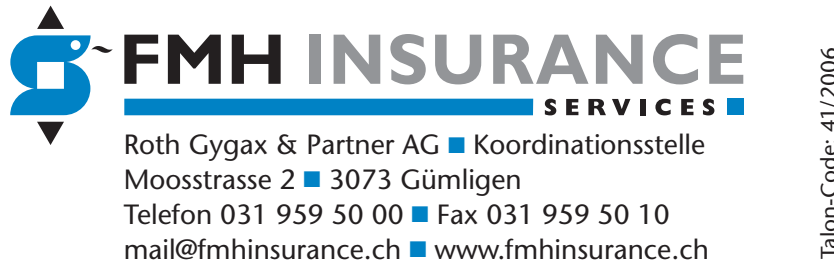

